\title{
Laser Two-Photon Ionization of 4,4"'-Bis[(2-butyloctyl)oxy]-p-quaterphenyl on a Platinum Surface in Ambient Air: Highly Sensitive Detection of Surface Molecules
}

\author{
Teiichiro OGawa*, To-oru YASUdA* and Hirofumi KaWAZUMI** \\ * Department of Molecular Science and Technology, Kyushu University, \\ Kasuga, Fukuoka 816, Japan \\ ** Division of Science, Kitakyushu University, Kokura-Minami, Kitakyushu 802, Japan
}

Keywords Laser, two-photon ionization, surface, 4,4"'-bis[(2-butyloctyl)oxy]-p-quaterphenyl, conductivity

Surface laser desorption and successive multiphoton ionization combined with mass spectrometry is a versatile and sensitive analytical technique ${ }^{1-4}$, which can be applied even to biological molecules. ${ }^{2,3}$ Like this method, however, most of the current techniques for highly sensitive detection on a surface require the sample to be in a high vacuum, even though there are many practical needs for surface analysis in ambient air.

The molecules on the surface emit photoelectrons upon optical irradiation, even in ambient air. ${ }^{5-7}$ Photoelectron spectrometry excited by a Xe lamp in ambient air has been applied for determining the work function of practical samples. ${ }^{8,9}$ However, a shortwavelength photon below $200 \mathrm{~nm}$ induces photoelectron emission very efficiently from almost any surface, and thus, the detection of a trace substance on a surface can not be carried out by using short-wavelength photons as an excitation source.

The present authors and their coworkers have studied analytical applications of laser two-photon ionization in solutions, and have obtained very high sensitivities. ${ }^{10,11}$ By using a two-photon technique, ionization of the solvent can be ignored and molecules with photoabsorption at the laser wavelength can be ionized efficiently and detected selectively. We have extended this technique to molecules on surfaces in ambient air. 4,4"'-Bis[(2-butyloctyl)oxy]-p-quaterphenyl (BBQ) on platinum is the first example of this approach to show its superiority.

\section{Experimental}

\section{Apparatus}

A schematic diagram of the experimental apparatus is shown in Fig. 1. The exciting radiation was provided by a Molectron UV24 nitrogen laser $(337 \mathrm{~nm}, 9 \mathrm{~mJ}$ /

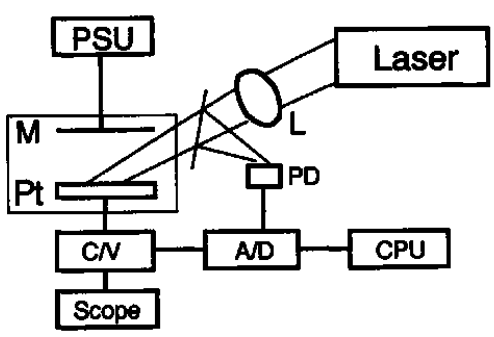

Fig. 1 Experimental apparatus. The platinum plate $(\mathrm{Pt})$ and the mesh electrode $(M)$ were placed in a shield box. The laser pulse energy was monitored with a pyroelectric detector (PD). PSU, power supply unit; $\mathrm{C} / \mathrm{V}$, current amplifier; A/D, AD converter; CPU, microcomputer; $L$, lens.

pulse); the laser beam was softly focused on the sample. A mesh electrode located $10 \mathrm{~mm}$ above the sample was connected to a high-voltage power supply unit (Ikegami HD2.5K-M); the typical applied voltage was $2.5 \mathrm{kV}$. The photocurrent signal was taken from the sample through a Keithley 427 current amplifier and a Canopus Analog Pro II analog-to-digital converter to an NEC 9801RX microcomputer. The time profile of the current signal was observed with a Iwatsu DS-6411 digital storage-scope; the time resolution was about $100 \mu \mathrm{s}$. The sample and the electrodes were kept in an aluminum box so as to remove any external noise.

\section{Sample preparation}

The sample was dispersed on a plate of platinum $(15 \times 15 \mathrm{~mm})$, which was polished with Maruto RDX K-1 celium oxide powder. Ten microliters of a hexane solution of BBQ was dropped onto the platinum plate, and dried in air. The sample was a thin layer of about $1 \mathrm{~cm}^{2}$, and was somewhat irregular. The laser radiation was irradiated on almost all of the sample surface so that 


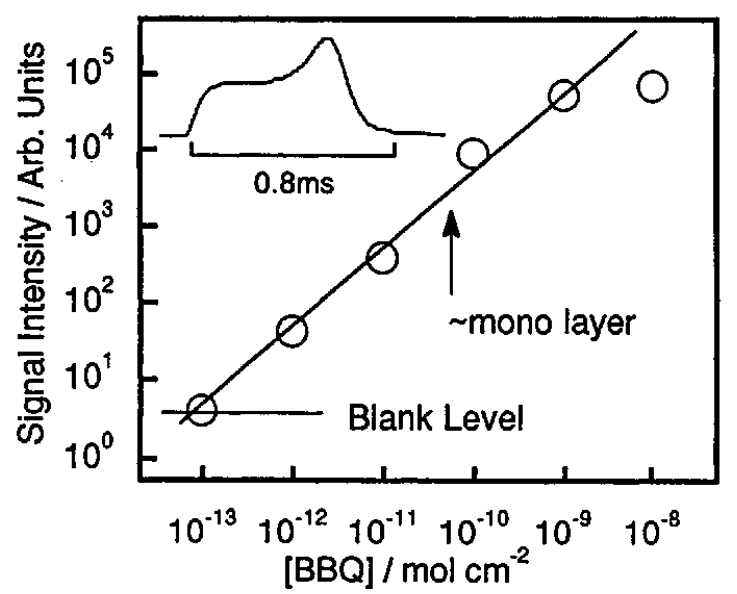

Fig. 2 Analytical curve and time profile of the photoionization current (insert) of BBQ on platinum excited by an $\mathrm{N}_{2}$ laser at $337 \mathrm{~nm}$. $\uparrow$, monolayer coverage; $0.8 \mathrm{~ms}$, timeregion for an area measurement.

the effect of non-uniformity of the sample could be reduced as much as possible. BBQ (Lambda Physik, laser dye) was used without further purification.

\section{Results and Discussion}

A typical time profile of the photocurrent of $B B Q$ on platinum is shown in Fig. 2. There were a fast, sharp component, and a slow, broad component, when the profile was measured at a faster time resolution. ${ }^{12} \mathrm{~A}$ slower time resolution, however, was used in the present study in order to increase the signal-to-noise ratio; the fast component apparently disappeared. The signal was almost unobservable when the mesh electrode was negatively biased.

The signal intensity was obtained by measuring the area of the photoionization current for $0-0.8 \mathrm{~ms}$ after the laser pulse, as shown in Fig. 2. For a quantitative measurement, signals for 400 laser pulses were accumulated in order to reduce any effect of fluctuation in laser pulse energy and to increase the signal-to-noise ratio. The laser pulse energy used was about $3 \mathrm{~mJ}$, and was softly focused onto the sample. If a higher pulse energy or a more focused beam was used, the sample was desorbed although the photocurrent increased. Laser pulse energy was kept so small that an effect of desorption was negligible within $\mathbf{4 0 0}$ pulses.

The photocurrent was proportional to the square of a laser pulse energy, indicating two-photon ionization.
The photocurrent was linearly proportional to the surface concentration of BBQ, as is also shown in Fig. 2. The blank level was the photocurrent of a bare platinum surface; it is either due to the photoelectric effect of the platinum surface or due to surface contamination in the laboratory atmosphere. The signal of a pure solvent was identical to that of the bare surface, indicating that any impurities left after evaporation were negligible. The analytical curve was linear for four orders of magnitude above the detection limit.

The detection limit of $\mathrm{BBQ}$ was estimated to be $2.5 \times 10^{-13} \mathrm{~mol} / \mathrm{cm}^{2}$ at $S / N=3$, which was about $1 / 200$ of the monolayer coverage. The monolayer coverage was estimated by assuming flat adsorption with a surface area of $3 \mathrm{~nm}^{2} /$ molecule. Although the conventional technique using a Xe lamp is useful for analyzing a bulk surface, it is not sensitive for a trace determination of surface molecules, because photoelectron emission from a bulk surface is very efficient. The two-photon technique can selectively ionize surface molecules which show the optical absorption at the laser wavelength.

Thus, the surface laser two-photon ionization technique has been found to be a very sensitive technique for trace molecules on a surface in ambient air, because the effects of photoelectron emission from a bulk surface can be removed.

\section{References}

1. R. Tembreull and D. M. Lubman, Anal. Chem., 59, 1003 (1987).

2. J. H. Hahn, R. Zenobi and R. N. Zare, J. Am. Chem. Soc., 109, 2842 (1987).

3. R. N. Zare, J. H. Hahn and R. Zenobi, Bull. Chem. Soc. Jpn., 61, 87 (1988).

4. B. Stahl, M. Steup, M. Karas and F. Hillenkamp, Anal. Chem., 63, 1463 (1991).

5. R. Naaman, A. Petrank and D. M. Lubman, J. Chem. Phys., 79, 4608 (1983).

6. R. B. Hall, J. Phys. Chem., 91, 1007 (1987).

7. K. R. Gopidas and P. V. Kamat, J. Phys. Chem., 93, 6428 (1989).

8. H. Kirihata and M. Uda, Rev. Sci. Instrum., 52, 68 (1981).

9. M. Shirahashi, M. Isobe and M. Uda, Denshi-Zairyo, 11, 1 (1985).

10. S. Yamada and T. Ogawa, Prog. Analyt. Spectrosc., 9, 429 (1986).

11. S. Yamada, N. Sato, H. Kawazumi and T. Ogawa, Anal. Chem., 59, 2719 (1987).

12. T. Yasuda, H. Kawazumi and T. Ogawa, to be published.

(Received October 10, 1991)

(Accepted December 19, 1991) 